\title{
BMJ Open Effect of an online healthy lifestyle psychoeducation programme to improve cardiometabolic outcomes and affective symptoms in youth receiving mental health care: study protocol for a pilot clinical trial.
}

Chloe Wilson (1) , Alissa Nichles (1) , Natalia Zmicerevska, Joanne Sarah Carpenter (1), Yun Ju Christine Song, Catherine McHugh (1) , Blake Hamilton, Samuel Hockey, Elizabeth M Scott, lan B Hickie

To cite: Wilson C, Nichles A, Zmicerevska N, et al. Effect of an online healthy lifestyle psychoeducation programme to improve cardiometabolic outcomes and affective symptoms in youth receiving mental health care: study protocol for a pilot clinical trial.. BMJ Open 2021;11:e044977. doi:10.1136/ bmjopen-2020-044977

- Prepublication history for this paper is available online. To view these files, please visit the journal online (http://dx.doi org/10.1136/bmjopen-2020044977).

Received 18 September 2020 Accepted 05 May 2021

Check for updates

(c) Author(s) (or their employer(s)) 2021. Re-use permitted under CC BY-NC. No commercial re-use. See rights and permissions. Published by BMJ.

Youth Mental Health and Technology Team, The University of Sydney Brain and Mind Centre, Sydney, New South Wales, Australia

Correspondence to

Chloe Wilson;

chloe.wilson@sydney.edu.au

\section{ABSTRACT}

Introduction Worsened cardiometabolic profiles in youth with mental ill health have been associated with a number of modifiable lifestyle risk factors. It is becoming increasingly evident that clinical interventions need to be multimodal in focus to improve mental health symptoms and the physical health symptoms in this already at-risk cohort.

Methods and analysis This 12-week pilot clinical trial examines the efficacy, feasibility and acceptability of an adjunctive online psychoeducation programme for improving cardiometabolic risk parameters and affective symptoms in a transdiagnostic sample of at least 44 young people aged 16-25 years presenting for mental healthcare for mood and/or psychotic syndromes (including anxiety, depression, bipolar disorder and psychosis). Individuals will be invited to participate in a pilot clinical trial for a structured online psychoeducation programme incorporating nutritional, physical activity, sleep-wake and healthy lifestyle information, delivered fortnightly over six online modules. Participants will undergo a series of assessments including: (1) self-report and clinician administered assessments determining mental health symptomatology; (2) fasting blood tests to assess cardiometabolic markers (fasting insulin, fasting glucose and blood lipids); (3) anthropometric assessments (height, weight, waist circumference and blood pressure); and (4) sleep-wake behaviours and circadian rhythm assessments. Changes in scores for all cardiometabolic and affective measures will be assessed via paired samples t-tests, and correlations between change scores will be assessed via Pearson's or Spearman's correlations. Feasibility will be assessed via completion rates, and the acceptability of the programme will be assessed via programme satisfaction measures.

Ethics and dissemination This pilot clinical trial has been approved by the Sydney Local Health District Research Ethics and Governance Office (X20-0228 \& 2020/ETH01201). The results of this pilot clinical trial will be disseminated into the scientific and broader community
Strengths and limitations of this study

- This pilot clinical trial evaluates a multidisciplinary online psychoeducation programme (involving structured nutrition, physical activity, sleep-wake and healthy lifestyle information) for improving cardiometabolic risk parameters and affective symptoms, in a transdiagnostic group of young people receiving mental healthcare.

- A range of cardiometabolic, anthropometric, sleepwake and mental health symptoms are assessed throughout the trial to determine whether these factors influence each other.

- Online delivery of psychoeducation modules will allow participants to access the information remotely.

- As this is a single-arm study, it cannot determine the efficacy of the intervention compared with other behavioural interventions in the same cohort.

- This pilot clinical trial will provide important information about how multidisciplinary behavioural modification programmes may help to manage the immediate cardiometabolic and affective symptoms of young people receiving mental healthcare; however, it cannot draw conclusions about the long-term effects of these lifestyle changes.

through peer-reviewed journals, conference presentations, social media and university websites.

Trial registration number Australian New Zealand Clinical Trials Registry (ANZCTR) Number: ACTRN12620000772943, Date 28 August 2020.

\section{BACKGROUND}

Premature mortality in those with severe mental illness has been well documented, ${ }^{1-6}$ with diabetes and premature cardiovascular disease comprising some of the leading causes of premature mortality and morbidity 
in this cohort. Increased body mass index (BMI) and weight gain have been identified as key modifiable risk factors causing premature mortality and morbidity in young people with severe mental illness, along with poor sleep-wake cycle regulation, ${ }^{7-9}$ physical inactivity, ${ }^{10-16}$ poor dietary habits ${ }^{10-20}$ and smoking. ${ }^{21-26}$

While many mental disorders can be managed with psychotherapy alone, psychotropic medications including antipsychotics, antidepressants and mood stabilisers are sometimes used for the treatment of a number of psychotic, affective and behavioural disorders in youth. ${ }^{27}$ Adolescents initiating psychotropic medications may display heterogeneous side effects with some very complex long-term cardiometabolic side effects. ${ }^{29-32}$ Of particular concern is the weight gain associated with second-generation antipsychotic usage, ${ }^{29}{ }^{33-37}$ which is acute and can often be observed within 12 weeks of medication initiation. ${ }^{38-43}$ Children and adolescents appear to be at a greater risk of psychotropic-induced weight gain than adults, ${ }^{44} 45$ and the younger the age of the child, the higher the mitigating risk. ${ }^{37}$ Psychotropic medication induced weight gain is a noteworthy adverse drug reaction as it mediates the development of other more severe cardiometabolic outcomes. ${ }^{38-43}$

Lifestyle interventions can be an effective alternative to pharmacological interventions to manage the physical and mental health symptoms of people presenting with psychiatric disorders. ${ }^{46}$ The World Health Organisation (WHO) guidelines recommend lifestyle behavioural interventions as a first-line treatment for physical health symptoms, including the cardiometabolic health, of adults with severe mental illness. ${ }^{47}$ The benefits of lifestyle and behavioural interventions for weight loss in obese individuals and for the prevention of type 2 diabetes mellitus are well established. ${ }^{48}{ }^{49}$ Additionally, psychoeducation interventions focusing on healthy lifestyle habits including nutrition, physical activity and sleep-wake practices have been shown to ameliorate both the physical and mental health concerns of young people with psychiatric disorders..$^{50-54}$

Face-to-face psychoeducation interventions for adults with severe mental illness are able to demonstrate significant improvements in weight $\mathrm{t}^{55}$; depressive symptoms, sleep quality and nutrition ${ }^{56}$; and other cardiometabolic risk factors. ${ }^{57}$ Existing web-based psychoeducation intervention studies on adults with mental illness have demonstrated improvements in depression and anxiety symptoms, ${ }^{58}$ with mixed evidence on the effect of psychotic symptoms. ${ }^{56} 59$ Other web-based psychoeducation studies on adults with mental illness have found increases in objective physical activity levels ${ }^{60}$; however, these studies have not measured cardiometabolic risk factors objectively. In youth, the literature in this field is more limited, with existing or planned studies in youth cohorts measuring affective symptom improvements only. ${ }^{61-63}$ Evidence on the effect of healthy lifestyle psychoeducation programmes on psychotic symptoms in youth is non-existent to our knowledge.
We are seeking to investigate the efficacy, feasibility and acceptability of a pilot clinical trial implementing an online healthy lifestyle psychoeducation programme targeted towards improving objective cardiometabolic outcomes and affective symptoms in young people presenting for mental healthcare for mood or psychotic syndromes (including anxiety, depression, bipolar disorder and/or psychosis). Web-based interventions provide an alternative to traditional face-to-face interventions. The need for online programmes is especially evident with the COVID-19 global pandemic impeding on young people's ability to seek face-to-face support. This has given rise to a need to develop more comprehensive resources tailored to the specific risk factors of this cohort in an easily accessible online format. Where effective, it would allow the programme to be implemented remotely to many individuals who may not be able to access in-person services.

\section{METHODS AND ANALYSIS}

\section{Patient and public involvement}

The study design, conduct and psychoeducation module content was developed in consultation with a representative from the Brain and Mind Centre Youth Lived Experienced Working Group. Patients or the public were not involved in the reporting, or dissemination plans of our research.

\section{Design and structure}

This is a one-arm longitudinal pilot clinical trial. The duration of the pilot clinical trial is 12 weeks. All participants will engage in an online 12-week psychoeducation programme. This psychoeducation programme will involve structured nutritional, physical activity, sleepwake and general healthy lifestyle information based on the Australian Guidelines of Physical Activity, the Australian Guide to Healthy Eating and published circadian research findings specific to youth mental illness. ${ }^{64-67}$ This information will be delivered for approximately 1 hour each fortnight over six online modules (week 1, 3, 5, 7, 9 and 11). The modules and study design have been developed in conjunction with mental health experts and those with a lived experience of mental ill health, specifically by presenting module material to a lived experience researcher and tailoring module content and delivery modes to ensure the suitability and relevance for this cohort. These modules are intensive and information dense, and by delivering these modules every 2 weeks, it allows the participants enough time to absorb the information and implement the advice into their lifestyle without becoming overwhelming.

Participants will be provided a link to access the online psychoeducation modules in their own time or given the opportunity to attend a live online or face-to-face module session. These modules will cover the topics shown in table 1.

Every week, participants will receive a monitoring phone call to aid the participant's engagement and ongoing 
Table 1 Psychoeducation modules

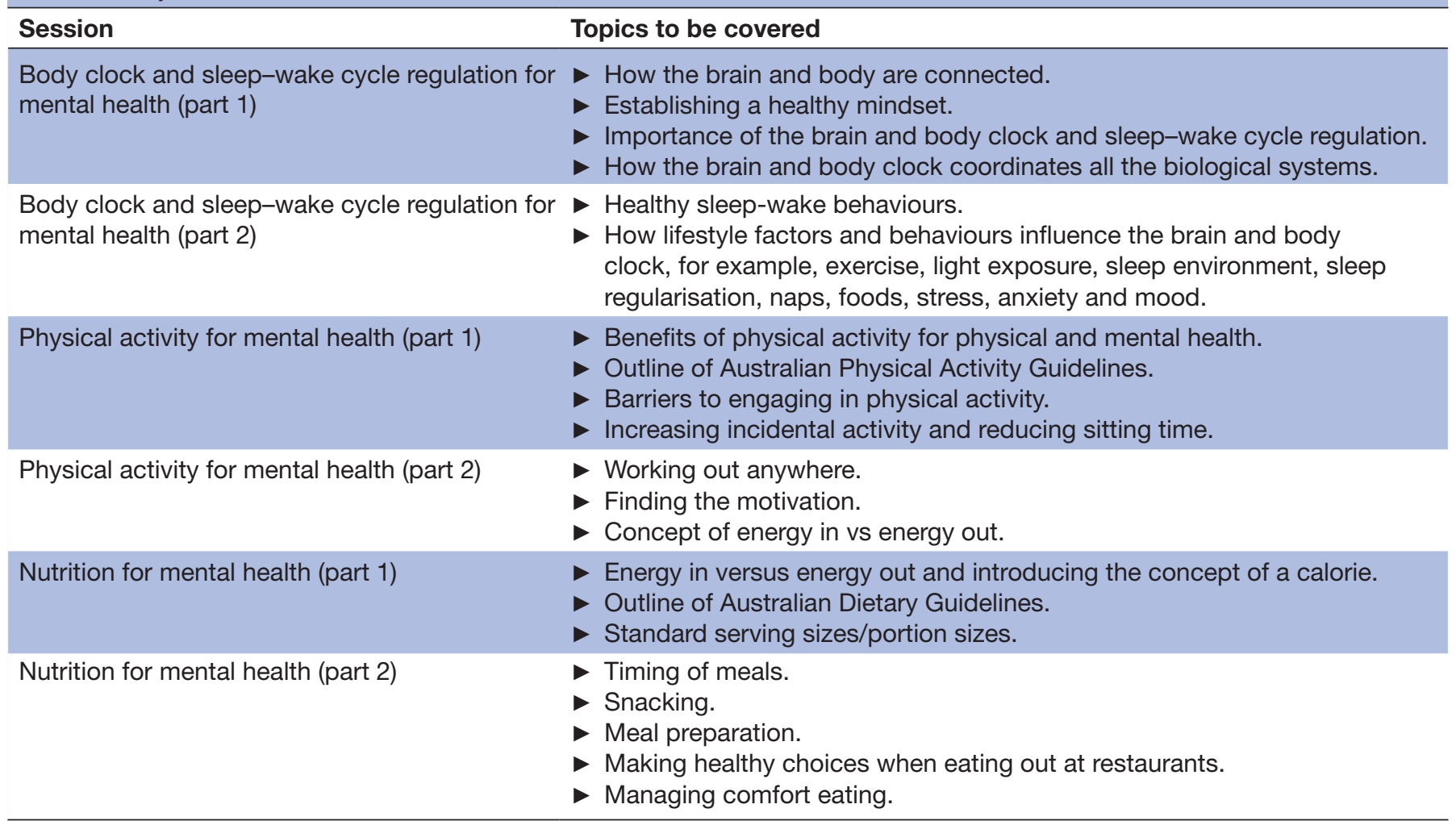

participation. This monitoring phone call will provide the participants with the opportunity to discuss the module content with the research staff, as well as discussing their goals and how the module content can be implemented into their lifestyle. At the completion of all six modules, the participant will be asked to provide feedback about the programme and to share their overall satisfaction with the programme via a series of online questionnaires. These questionnaires will provide important information about the acceptability and feasibility of the psychoeducation programme in this cohort.

Participants will be asked to wear an actigraph (GENEActiv; Activinsights, Kimbolton, UK) on the non-dominant wrist to collect 24-hour sleep-wake and physical activity parameters during weeks 1-2, 6-7 and 11-12. Fasting blood tests to measure metabolic markers; anthropometric assessments (blood pressure, height, weight and waist circumference); self-report and clinician administered assessments to assess various mental illness symptoms; and physical activity engagement will be conducted in weeks 1 and 12. All assessments including the selfreport questionnaires and clinician rated assessments are expected to take approximately 2 hours at each time point.

Most of these self-report and clinician administered assessments are part of the standardised assessment battery developed for the Youth Mental Health Tracker as part of the Brain and Mind Centre (BMC) multidimensional research framework. ${ }^{68}$ The multidimensional research framework was developed to assess a comprehensive range of measures in individuals presenting to care across a range of domains important to mental health outcomes. All observational and interventional youth mental health research at the BMC uses a standardised set of measures within this framework. These assessments are part of an ongoing larger study for all young people presenting for mental healthcare to improve the outcomes of their clinical care. Other clinician administered measures have been included in this study as they are improved and updated measures of self-report measures that are subject to bias and reporting errors.

The schedule of enrolment, interventions and assessment time points can be seen in table 2 .

\section{Setting, recruitment and informed consent}

This is a single-site, transdiagnostic intervention study, conducted at the BMC (including headspace, Camperdown, and Early Intervention and High Intensity Services) at the University of Sydney (Sydney, Australia).

Potential participants will be identified as young people aged between 16 and 25 years of age presenting for mental healthcare, who are at risk for poor cardiometabolic outcomes due to being overweight or obese. To avoid recruitment bias, all treating clinicians will be made aware of the study and eligibility criteria and will encourage all suitable young people presenting for care at these services to participate in the study.

With the young person's permission, the referring clinicians will pass on the contact details to the study research team, who will make further contact and initiate the informed consent process. The research team will make explicit to any potential participants both verbally and 
Table 2 Schedule of enrolment, interventions and assessment time points

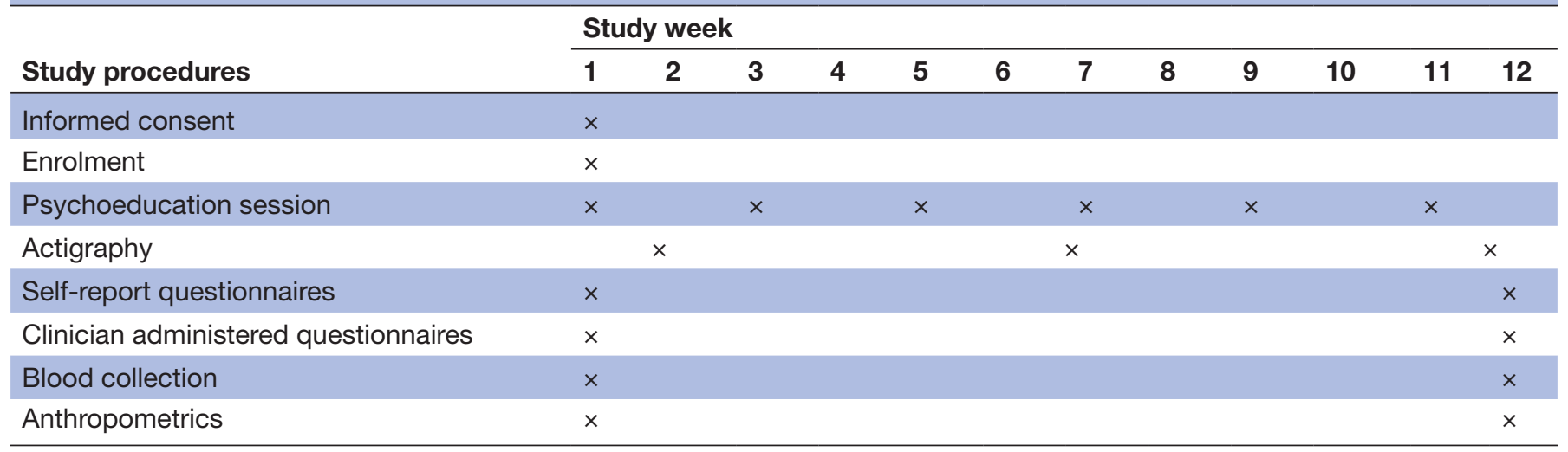

in writing (in the participant information and consent form) that participation is voluntary. The study staff will then obtain written informed consent from the young person to participate in the psychoeducation programme. Each participant will have a participant ID that links the participant to the research database in a confidential and deidentified manner for the purpose of research.

Participants are free to withdraw from the study at any time by contacting research staff. They will be assured that their decision whether to participate will not affect their current or future relationship with the researchers or anyone else at The University of Sydney nor their current or future involvement with the mental health service. If participants withdraw from the study, all future data (ie, from thereon) will not be stored or used for research purposes.

\section{Selection criteria}

Young people will be invited into the trial based on the following inclusion criteria: (1) aged between 16 and 25 years; (2) receiving mental healthcare treatment at the participating sites; (3) BMI $\geq 23$; and (4) willing and able to give independent written informed consent to participate in the study.

The exclusion criteria are: (1) intellectual disability (at the discretion of a clinical psychologist or psychiatrist); (2) major neurological disorder, medical illness that impacts on cognition and/or a history of sustained head injury; (3) not fluent in English; or (4) an acute psychotic or manic episode that impairs the individual's ability to give informed consent and/or requires acute clinical treatment.

\section{Study objectives}

\section{Primary}

To assess the efficacy of an online 12-week healthy lifestyle psychoeducation programme in improving cardiometabolic parameters (fasting insulin, fasting glucose, the updated homeostatic model assessment (HOMA2-IR), cholesterol, triglycerides, blood pressure, BMI and waist circumference) of young people seeking treatment for mental health related issues.

\section{Secondary}

To assess the efficacy of an online 12-week healthy lifestyle psychoeducation programme in improving affective (depressive and anxiety) symptoms of young people seeking treatment for mental health-related issues.

\section{Tertiary}

To assess if changes in cardiometabolic health parameters (fasting insulin, fasting glucose, HOMA2-IR, cholesterol, triglycerides, blood pressure, BMI and waist circumference) are associated with changes in affective (depressive and anxiety) symptom severity.

To assess the feasibility and acceptability of the psychoeducation programme in a cohort of young people seeking treatment for mental health related issues.

\section{Measures}

Key outcome measures targeted for this study are highlighted in italic type.

\section{CLINICIAN-RATED ASSESSMENTS}

1. Diagnostic assessment: the presence of any Diagnostic and Statistical Manual of Mental Disorders (DSM-V) disorders will be assessed using the Structured Clinical Interview for DSM-V Disorders. ${ }^{69}$

2. Physical health, mental health, family health and treatment history: current and past health history will be assessed and recorded by trained researchers and study doctors. This includes current medication and any changes in physical and/or mental health treatment being received throughout the trial.

3. The Brief Psychiatric Rating Scale $(B P R S)^{70}$ : the BPRS measures psychiatric symptoms including depression, anxiety, hallucinations, delusions and unusual behaviour. This 24-item scale is one of the most universally used scales to measure psychotic symptoms. ${ }^{71}$

4. Clinical staging ${ }^{72}$ : this framework stages individuals according to the presentation of their mental illness from those in the earliest phases with non-specific clinical presentations (stages 1a 'seeking help'), those at greater risk with more specific, subthreshold 
presentations (stage 1b 'attenuated syndromes') and those who have already reached a threshold for a progressive or recurrent disorder meeting diagnostic criteria (stages 2,3 or 4$){ }^{72-75}$

5. Pathophysiological mechanisms ${ }^{76}$ : the pathophysiological model suggests three possible pathways of illness: (1) neurodevelopmental impairments, (2) circadian dysregulation and (3) heightened sensitivity (ie, stress-reactivity) in the 'fear' circuitry. Based on these pathways, three clinical phenotype labels will be assigned to the individual: (1) developmental psychotic, (2) mania-fatigue or (3) anxiety depression subtypes.

6. Clinical Global Impression (CGI $)^{77}$ : the CGI provides an indication of the young person's ability to function in context of their history, psychosocial circumstances, symptoms and behaviour. The CGI comprises two one-item measures evaluating the severity of psychopathology from 1 to 7 (CGI-Severity (CGI-S) ) and the change from the initiation of treatment on a seven-point scale (CGI-Improvement (CGI-I)).

7. The Young Mania Rating Scale $(Y M R S)^{78}$ : the YMRS is an 11-item questionnaire measuring manic episode severity derived from the young person's subjective report and clinical observations during the clinical interview.

8. The Simple Physical Activity Questionnaire $(\operatorname{SIMPAQ})^{79}$ : the SIMPAQ is a five-item clinical tool designed to assess the degree of physical activity in cohorts at high risk of sedentary behaviour. This measure has been used in over 23 countries and can be reliability and validly administered by clinicians. ${ }^{80}$

9. Suicidal ideation and behaviour: acute suicidal behaviour will be assessed by item 7.3 of the Comprehensive Assessment of At-Risk Mental States (CAARMS). ${ }^{81}$ The CAARMS is a semistructured assessment tool designed to identify those at ultra-high risk for psychosis. This assessment is only to be administered as a safety measure where the self-report Suicidal Ideation Attributes Scale (SIDAS) score reaches the cut-off score of $\geq 21$ indicating a high risk of suicidal behaviour.

10. Social and Occupational Assessment Scale (SOFAS): ${ }^{38}$ The SOFAS is a global rating of the participant's current social and occupational functioning, independent of the overall severity of the individual's psychological symptoms, ranging from 0 to 100 , with lower scores indicating poorer functioning. The SOFAS has been used extensively in clinical research and practice and has good construct validity, inter-rater reliability and predictive validity. ${ }^{82} 83$

\section{SELF-REPORT QUESTIONNAIRES}

1. Demographics: this questionnaire will comprise details on basic demographics (including details of work and education, ethnicity, living circumstances and relationship status) and physical health (height, weight and waist circumference).

2. Kessler Psychological Distress Scale $(K-10)^{84}{ }^{85}$ : the K-10 is a 10-item version of the Kessler scale that provides a global measure of distress derived from anxiety and depressive symptoms over a 4-week period. It has high predictive validity and reliability. ${ }^{86}$

3. International Physical Activity Questionnaire (IPAQ) short version ${ }^{87}{ }^{88}$ : the short version of the IPAQ is a seven-item questionnaire calculating the amount of time spent engaging in sedentary activity, and mild, moderate, or vigorous-intensity physical activity. The original IPAQ has been tested across populations and broad age groups and demonstrates good reliability and validity. ${ }^{87}$

4. Somatic and Psychological Health Report (SPHERE 12) ${ }^{89}$ : The SPHERE 12 is a 12-item self-report questionnaire that assesses six psychological (PSYCH subscale), and six physical and somatic/fatigue symptoms (SOMA subscale) to identify anxiety, depression and somatisation symptoms in primary care. The SPHERE-12 has been demonstrated to have high reliability and validity in populations over the age of 16 years. ${ }^{89}$

5. Sleep-wake cycle and chronotype: six questions will be asked concerning time falling asleep, time waking up during weekdays and weekends, hours of sleep and feelings when waking up. Sleep timing items are based on the Pittsburgh Sleep Quality Index (PSQI) ${ }^{90}$ and Munich Chrono Type Questionnaire, ${ }^{91}$ while sleep quality items were developed based on expert agreement in existing literature.

6. $P S Q I^{90}$ : the PSQI is a 24-item self-report questionnaire measuring the quality and patterns of sleep. Seven domains are assessed including subjective sleep quality, sleep latency, sleep duration, habitual sleep efficiency, sleep disturbances, use of sleeping medications and daytime dysfunction over the last month. This scale has good internal consistency and construct validity. ${ }^{92} 93$

7. The Insomnia Severity Index ${ }^{94}$ : this seven-item questionnaire assesses the severity of difficulties in initiating sleep, staying asleep, early morning awakenings, satisfaction with current sleep pattern, daily functioning and impairment attributed to the sleep problem, and the degree of distress or concern caused by the sleep problem. This scale has been used widely in clinical research with high internal consistency and convergent validity. ${ }^{95}$

8. SIDAS ${ }^{96}$ : the SIDAS is a five-item self-report questionnaire assessing suicidal ideology in relation to the frequency, controllability, closeness to attempt, distress and interference with daily activities over the last month. The SIDAS has demonstrated high internal consistency and good convergent validity. ${ }^{96}$

9. Quick Inventory of Depressive Symptomatology - self-report $(\text { QIDS-SR })^{97}$ : The QIDS is a rating scale assessing nine criterion symptom domains (sleep, sad mood, appetite/weight, concentration/decision making, 
self-view, thoughts of death or suicide, general interest, energy level and restlessness/agitation) outlined by the DSM-IV to diagnose a major depressive episode. This scale has high internal consistency and good predictive and concurrent validity. ${ }^{97}$

10. Overall Anxiety Severity Impairment Scale $(O A S I S)^{98}$ : the OASIS is a five-item self-report measure used to assess the frequency and intensity of anxiety symptoms, including the functional impairment and behavioural avoidance commonly associated with any anxiety disorder or multiple anxiety disorders. This scale has excellent test-retest reliability, and convergent and discriminant validity. ${ }^{98}$

11. WHO Alcohol, Smoking and Substance Involvement Screening Test (WHO-ASSIST) 9910099100 : the ASSIST (V.3.1) is an eight-item questionnaire screening for use of tobacco products, alcohol, cannabis, cocaine, amphetamine-type stimulants, sedatives and sleeping pills (benzodiazepines), hallucinogens, inhalants, opioids and 'other' drugs. This scale has demonstrated good validity ${ }^{101}$ and reliability. ${ }^{100}$

12. Alcohol Use Disorders Identification Test Consumption (AUDIT-C) ${ }^{102}$ : the AUDIT-C is a three-item scale gauging alcohol consumption in a standardised manner. This scale has shown good reliability and validity in adolescent sample groups. ${ }^{103}$

13. Eating Disorder Examination ${ }^{104}{ }^{105}$ : this self-report questionnaire assesses current eating disorder behaviours, including binge eating, purging and strict dieting or fasting. This self-report questionnaire has demonstrated good internal consistency and construct validity. ${ }^{106}$

14. Rosenberg Self-Esteem Scale (RSES $)^{107}$ : the RSES is a 10item self-report measure of self-esteem, self-worth or self-acceptance designed specifically for use in adolescent populations. Higher scores on this scale indicate higher levels of global self-esteem. This scale has extremely high internal consistency and demonstrated construct validity with other measures of selfesteem. ${ }^{108} 109$

15. Client Satisfaction Questionnaire-8 $(C S Q-8)^{110}$ : the CSQ assesses level of satisfaction with care and has been found to have high internal consistency and concurrent validity in mental health outpatient settings. ${ }^{110}$

16. Feedback questionnaire: this is an investigator-developed questionnaire specifically relating to the feasibility and acceptability of the psychoeducation programme and whether the participants have any feedback or suggestions for improvement to the online psychoeducation programme after completion of the programme.

\section{Blood markers}

Blood samples are to be collected in a fasting state between 08:00 and 10:00 by a trained phlebotomist at week 1 and week 12 to determine variables of interest including fasting glucose, fasting insulin and blood lipids (including triglycerides, total cholesterol, high density lipoprotein cholesterol and low-density lipoprotein cholesterol levels). Other blood measures to be collected for monitoring purposes include $H b A 1 c$, full blood count, urea, electrolytes, liver function test, C reactive protein, erythrocyte sedimentation rate, antinuclear antibodies, vitamin $\mathrm{D}$, vitamin B12, folate, iron, thyroid stimulating hormone, calcium, magnesium and phosphate levels. Insulin resistance will be estimated using the updated homeostatic model assessment (HOMA2-IR) using iHOMA2 software V.8.8 ${ }^{111}$ from fasting blood test results. Metabolic blood measures will be collected by a standard pathology request, and a deidentified copy of the results will be kept in the participants' file.

\section{Anthropometric assessments}

Measures of blood pressure, height and weight will be collected via direct measurement by a clinician or research staff. $B M I$ will be calculated using the formula: weight $(\mathrm{kg}) \div$ height $(\mathrm{m})^{2}$. Waist circumference is measured with the participant standing up, to the nearest $1 \mathrm{~cm}$ with a measuring tape at the midpoint between the bottom of the rib cage and above the top of the iliac crest (hip bone) at the end of the participant's normal respiration. Where the young person is unable to visit the BMC for an anthropometric assessment, the participant will be required to self-report height and weight and will be instructed how to gather waist circumference measurements.

\section{4-hour sleep-wake and physical activity profiling}

All participants will wear wrist mounted actigraphy recording devices (GENEActiv Sleep device; Activinsights, Kimbolton, UK) to record motor activity over a 2-week period for an estimation of sleep and physical activity patterns based on validated algorithms. Measurements include sleep onset time, sleep offset time, sleep midpoint, sleep efficiency, wake after sleep onset (number of minutes during the sleep period scored as awake) and total sleep time (number of minutes during the sleep period scored as sleep).

Physical activity levels will be assessed through the GENEActiv devices as gross motor activity per day (milligravity $\left.(\mathrm{mg}), 1 \mathrm{~g}=9.81 \mathrm{~m} / \mathrm{s}^{2}\right)$ and minutes of moderate-tovigorous physical activity per day (defined as the sum of 1 min epochs in which gross motor activity is larger than $125 \mathrm{mg}$ ) as described in other studies. ${ }^{112}$ The GENEActiv devices have been used widely in clinical research and validated against several types of accelerometry-based activity monitors ${ }^{113-116}$ as well as for sleep-wake scoring. ${ }^{117} 118$

\section{Sample size/power calculation}

The proposed sample size calculation of at least 44 participants is based on specific power analysis parameters (power analyses completed in $G^{*}$ Power V.3.1.9.4), notably difference in means in HOMA2-IR scores of one sample with a power of 0.90 , an effect size of 0.5 and an alpha level of 0.05 . While this is a pilot study, and a sample size has been estimated, these pilot data will be used to inform power calculations for future studies investigating similar outcome measures in this specific cohort. 


\section{Data analysis plan}

All data will be entered into a secure password-protected database and statistical analyses conducted in $\mathrm{R}$ statistical software. The primary and secondary objectives will be analysed by a change in mean scores of all outcome measures after completion of the 12-week online psychoeducation programme via a paired samples t-test, with significance levels set at $\alpha=0.05$. To assess the tertiary objectives, correlations will be performed between all cardiometabolic change scores and affective outcome change scores via Pearson's or Spearman's correlations tests based on normative or non-normative data distribution, with a significance level set at $\alpha=0.05$. Overall completion rates of the study will be used to assess feasibility, and the average of the CSQ- 8 scores will be used to assess acceptability.

\section{Ethics and dissemination}

The Sydney Local Health District (RPAH Division) Human Ethics Research Committee has approved this study (X20-0228). The online psychoeducation programme is designed as an adjunct, not an alternative, to standard clinical treatments offered by the youth mental health services. As such, all participants are encouraged to continue to follow the healthcare advice of their treating clinicians and to remain in their care, as well as participating in the online psychoeducation sessions. This standard treatment may include medication, counselling, psychological therapy and/or referrals to a range of specialist mental health treatments or services.

The headspace, Camperdown clinic is an established, specialised clinic for the youth mental health population. As such, the clinic has its own internal crisis management plans and are staffed with leading expertise in managing distress and unexpected occurrences for the youth mental health population. As a further precaution, questions about suicidal ideation or self-harm will be asked in weeks $1,4,8$ and 12 in the monitoring phone call. If there is an indication of suicidal intent, the CAARMS will be administered and suicidality standard operating protocol will be followed. Any adverse events will be logged into an adverse event monitoring log.

In accordance with the guidelines stipulated by the funding bodies, the results of this study will be disseminated as widely as possible into the scientific and broader community. This may include publication in peer-reviewed journals, scholarly book chapters, presentation at conferences and publication in conference proceedings. In accordance with the National Health and Medical Research Council policy, publications arising from this study will be deposited into an open access institutional repository, where possible. It is also intended for results to be disseminated into the wider community in a format appropriate for a lay audience, through links including the BMC website and social media, as well as newsletters.

\section{DISCUSSION}

This pilot clinical trial plans to evaluate the efficacy, feasibility and acceptability of a 12-week online psychoeducation programme (involving structured nutrition, physical activity, sleep-wake and healthy lifestyle information) on several cardiometabolic risk factors and affective symptoms in youth seeking treatment for mental ill health. The multidimensional modules feature a youth-friendly format, with input from those with lived experience, to maximise engagement by young people. If the findings of this pilot clinical trial demonstrate benefits in each of the targeted domains, it will be used as a basis for further clinical trials incorporating more intensive psychoeducation combined with pharmacological therapies. Due to the online nature of the psychoeducation programme, there is also potential for a larger scale study to be implemented across a number of centres nationally, allowing individuals to access the programme remotely and regionally.

While this pilot study will provide important information about how multidisciplinary behavioural modification programmes may help to manage the immediate cardiometabolic and mental health symptoms (including affective, anxiety and psychotic symptoms) of young people receiving mental healthcare, it cannot draw conclusions about the long-term implications of these lifestyle changes on the assessed domains. Additionally, as this is a single-arm pilot study, it cannot determine the efficacy of the intervention compared with other behavioural interventions in the same cohort. Future research should thus aim to monitor participants over longer periods of time while comparing to other pharmacological (eg, metformin) or behavioural interventions.

\section{Trial status \\ The trial has begun recruitment.}

Contributors $\mathrm{CW}$ has developed this clinical trial as part of her $\mathrm{PhD}$ research project and drafted this original manuscript with input from other authors. IBH assisted with the design of the study. AN, NZ, JSC, YJCS, CM, BH, SH and EMS were all involved with modifications to the design of the study and with drafting of this paper. All authors have read and approved the final manuscript.

Funding This project is an investigator-initiated study, sponsored by the University of Sydney and supported by philanthropic funding, for which donor(s) are families affected by mental illness who wish to remain anonymous. This study was also partially funded by a philanthropic PhD scholarship (The Liu McCabe Family Scholarship awarded to CW), a National Health \& Medical Research Council Australia Fellowship (No. 511921, awarded to IBH) and a philanthropic fellowship (the Caroline Quinn Research Grant awarded to JC).

Disclaimer The funders of this study had no involvement in the: study design; collection, analysis and reporting of the data; writing of the report; or decision to submit the paper for publication.

Competing interests Professor lan Hickie was an inaugural Commissioner on Australia's National Mental Health Commission (2012-18). He is the Co-Director, Health and Policy at the Brain and Mind Centre (BMC) University of Sydney. The $\mathrm{BMC}$ operates early-intervention youth services at Camperdown under contract to headspace. He is the Chief Scientific Advisor to, and a 5\% equity shareholder in, InnoWell Pty LTd. InnoWell was formed by the University of Sydney (45\% equity) and PwC (Australia; $45 \%$ equity) to deliver the \$30M Australian Government-funded Project Synergy (2017-20; a three-year program for the transformation of mental health services) and to lead transformation of mental health services internationally 
through the use of innovative technologies.A/Prof Elizabeth Scott is Principal Research Fellow at the Brain and Mind Centre, The University of Sydney. She is Discipline Leader of Adult Mental Health, School of Medicine, University of Notre Dame, and a Consultant Psychiatrist. She was the Medical Director, Young Adult Mental Health Unit, St Vincent's Hospital Darlinghurst until January 2021. She has received honoraria for educational seminars related to the clinical management of depressive disorders supported by Servier and Eli-Lilly pharmaceuticals. She has participated in a national advisory board for the antidepressant compound Pristiq, manufactured by Pfizer. She was the National Coordinator of an antidepressant trial sponsored by Servier.

Patient and public involvement Patients and/or the public were involved in the design, or conduct, or reporting, or dissemination plans of this research. Refer to the Methods section for further details.

Patient consent for publication Not required.

Provenance and peer review Not commissioned; externally peer reviewed. Data availability statement Not required

Open access This is an open access article distributed in accordance with the Creative Commons Attribution Non Commercial (CC BY-NC 4.0) license, which permits others to distribute, remix, adapt, build upon this work non-commercially, and license their derivative works on different terms, provided the original work is properly cited, appropriate credit is given, any changes made indicated, and the use is non-commercial. See: http://creativecommons.org/licenses/by-nc/4.0/.

\section{ORCID iDs}

Chloe Wilson http://orcid.org/0000-0001-6539-423X

Alissa Nichles http://orcid.org/0000-0001-6404-7199

Joanne Sarah Carpenter http://orcid.org/0000-0002-9766-6700

Catherine McHugh http://orcid.org/0000-0002-4891-4966

\section{REFERENCES}

1 Liu NH, Daumit GL, Dua T, et al. Excess mortality in persons with severe mental disorders: a multilevel intervention framework and priorities for clinical practice, policy and research agendas. World Psychiatry 2017;16:30-40.

2 Chesney E, Goodwin GM, Fazel S. Risks of all-cause and suicide mortality in mental disorders: a meta-review. World Psychiatry 2014;13:153-60.

3 Laursen TM, Musliner KL, Benros ME, et al. Mortality and life expectancy in persons with severe unipolar depression. $J$ Affect Disord 2016;193:203-7.

4 Cuijpers P, Vogelzangs N, Twisk J, et al. Comprehensive meta-analysis of excess mortality in depression in the general community versus patients with specific illnesses. Am J Psychiatry 2014:171:453-62.

5 Hayes JF, Miles J, Walters K, et al. A systematic review and metaanalysis of premature mortality in bipolar affective disorder. Acta Psychiatr Scand 2015;131:417-25.

6 Roberts LW, Louie AK, Guerrero APS, et al. Premature mortality among people with mental illness: advocacy in academic psychiatry. Acad Psychiatry 2017;41:441-6.

7 Cespedes Feliciano EM, Quante M, Rifas-Shiman SL, et al. Objective sleep characteristics and cardiometabolic health in young adolescents. Pediatrics 2018;142:e20174085.

8 Quante M, Cespedes Feliciano EM, Rifas-Shiman SL, et al. Association of daily Rest-Activity patterns with adiposity and cardiometabolic risk measures in teens. J Adolesc Health 2019:65:224-31.

9 Grandner MA, Jackson NJ, Pak VM, et al. Sleep disturbance is associated with cardiovascular and metabolic disorders. J Sleep Res 2012;21:427-33.

10 Ringen PA, Faerden A, Antonsen B, et al. Cardiometabolic risk factors, physical activity and psychiatric status in patients in long-term psychiatric inpatient departments. Nord J Psychiatry 2018;72:296-302.

11 Scheewe TW, Jörg F, Takken T, et al. Low physical activity and cardiorespiratory fitness in people with schizophrenia: a comparison with matched healthy controls and associations with mental and physical health. Front Psychiatry 2019;10:87.

12 Vancampfort D, Stubbs B. Physical activity and metabolic disease among people with affective disorders: prevention, management and implementation. J Affect Disord 2017;224:87-94.

13 Vancampfort D, Firth J, Schuch F, et al. Physical activity and sedentary behavior in people with bipolar disorder: a systematic review and meta-analysis. J Affect Disord 2016;201:145-52.
14 Vancampfort D, Firth J, Schuch FB, et al. Sedentary behavior and physical activity levels in people with schizophrenia, bipolar disorder and major depressive disorder: a global systematic review and meta-analysis. World Psychiatry 2017;16:308-15.

15 Schuch F, Vancampfort D, Firth J, et al. Physical activity and sedentary behavior in people with major depressive disorder: a systematic review and meta-analysis. J Affect Disord 2017;210:139-50.

16 Vancampfort D, Sienaert P, Wyckaert S, et al. Sitting time, physical fitness impairments and metabolic abnormalities in people with bipolar disorder: an exploratory study. Psychiatry Res 2016;242:7-12.

17 Soreca I, Wallace ML, Hall MH, et al. The association between mea timing and frequency with cardiometabolic profile in patients with bipolar disorder. Acta Psychiatr Scand 2016;133:453-8.

18 Teasdale SB, Ward PB, Samaras K, et al. Dietary intake of people with severe mental illness: systematic review and meta-analysis. $\mathrm{Br}$ $J$ Psychiatry 2019;214:251-9.

19 Firth J, Stubbs B, Teasdale SB, et al. Diet as a hot topic in psychiatry: a population-scale study of nutritional intake and inflammatory potential in severe mental illness. World Psychiatry 2018;17:365-7.

20 Barker MK, Sable CM, Montgomery SE, et al. Diet and cardiometabolic side effects in children treated with secondgeneration antipsychotics. Clin Nutr ESPEN 2018;23:205-11.

21 Mallet J, Le Strat Y, Schürhoff F, et al. Tobacco smoking is associated with antipsychotic medication, physical aggressiveness, and alcohol use disorder in schizophrenia: results from the FACE-SZ national cohort. Eur Arch Psychiatry Clin Neurosci 2019;269:449-57.

22 Jackson JG, Diaz FJ, Lopez L, et al. A combined analysis of worldwide studies demonstrates an association between bipolar disorder and tobacco smoking behaviors in adults. Bipolar Disord 2015;17:575-97.

23 Richardson S, McNeill A, Brose LS. Smoking and quitting behaviours by mental health conditions in Great Britain (1993-2014). Addict Behav 2019;90:14-19.

24 Prochaska JJ, Das S, Young-Wolff KC. Smoking, mental illness, and public health. Annu Rev Public Health 2017;38:165-85.

25 Callaghan RC, Veldhuizen S, Jeysingh T, et al. Patterns of tobaccorelated mortality among individuals diagnosed with schizophrenia, bipolar disorder, or depression. J Psychiatr Res 2014;48:102-10.

26 Weinberger AH, Streck JM, Pacek LR. Nondaily cigarette smoking is increasing among people with common mental health and substance use problems in the United States: data from representative samples of US adults, 2005-2014. J Clin Psychiatry 2018;79.

27 Seida JC, Schouten JR, Boylan K, et al. Antipsychotics for children and young adults: a comparative effectiveness review. Pediatrics 2012;129:e771-84

28 Cheng-Shannon J, McGough JJ, Pataki C, et al. SecondGeneration antipsychotic medications in children and adolescents. $J$ Child Adolesc Psychopharmacol 2004;14:372-94.

29 Pillay J, Boylan K, Carrey N. First-and second-generation antipsychotics in children and young adults: systematic review update 2017.

30 Jerrell JM, Mclntyre RS. Adverse events in children and adolescents treated with antipsychotic medications. Hum Psychopharmacol 2008:23:283-90.

31 De Hert M, Yu W, Detraux J, et al. Body weight and metabolic adverse effects of asenapine, iloperidone, lurasidone and paliperidone in the treatment of schizophrenia and bipolar disorder: a systematic review and exploratory meta-analysis. CNS Drugs 2012;26:733-59.

32 Ngai YF, Sabatini P, Nguyen D, et al. Quetiapine treatment in youth is associated with decreased insulin secretion. $J$ Clin Psychopharmacol 2014;34:359-64.

33 Panagiotopoulos C, Ronsley R, Davidson J. Increased prevalence of obesity and glucose intolerance in youth treated with second-generation antipsychotic medications. Can J Psychiatry 2009;54:743-9.

34 Scott EM, Hermens DF, White D, et al. Body mass, cardiovascular risk and metabolic characteristics of young persons presenting for mental healthcare in Sydney, Australia. BMJ Open 2015;5:e007066.

35 Fraguas D, Correll CU, Merchán-Naranjo J, et al. Efficacy and safety of second-generation antipsychotics in children and adolescents with psychotic and bipolar spectrum disorders: comprehensive review of prospective head-to-head and placebo-controlled comparisons. Eur Neuropsychopharmacol 2011;21:621-45.

36 Zuddas A, Zanni R, Usala T. Second generation antipsychotics (SGAs) for non-psychotic disorders in children and 
adolescents: a review of the randomized controlled studies. Eur Neuropsychopharmacol 2011;21:600-20.

37 Martínez-Ortega JM, Funes-Godoy S, Díaz-Atienza F, et al. Weight gain and increase of body mass index among children and adolescents treated with antipsychotics: a critical review. Eur Child Adolesc Psychiatry 2013;22:457-79.

38 Baeza I, Vigo L, de la Serna E, et al. The effects of antipsychotics on weight gain, weight-related hormones and homocysteine in children and adolescents: a 1-year follow-up study. Eur Child Adolesc Psychiatry 2017;26:35-46.

39 Stigler KA, Potenza MN, Posey DJ, et al. Weight gain associated with atypical antipsychotic use in children and adolescents. Pediatric Drugs 2004;6:33-44.

40 De Hert M, Dobbelaere M, Sheridan EM, et al. Metabolic and endocrine adverse effects of second-generation antipsychotics in children and adolescents: a systematic review of randomized placebo controlled trials and guidelines for clinical practice. Eur Psychiatry 2011;26:144-58.

41 Correll CU, Carlson HE. Endocrine and metabolic adverse effects of psychotropic medications in children and adolescents. J Am Acad Child Adolesc Psychiatry 2006;45:771-91.

42 Maayan L, Correll CU. Weight gain and metabolic risks associated with antipsychotic medications in children and adolescents. J Child Adolesc Psychopharmacol 2011;21:517-35.

43 Correll CU, Manu P, Olshanskiy V, et al. Cardiometabolic risk of second-generation antipsychotic medications during first-time use in children and adolescents. JAMA 2009;302:1765-73.

44 Arango C, Robles O, Parellada M, et al. Olanzapine compared to quetiapine in adolescents with a first psychotic episode. Eur Child Adolesc Psychiatry 2009;18:418-28.

45 Safer DJ. A comparison of risperidone-induced weight gain across the age span. $J$ Clin Psychopharmacol 2004;24:429-36.

46 Briguglio M, Vitale JA, Galentino R, et al. Healthy eating, physical activity, and sleep hygiene (HEPAS) as the winning triad for sustaining physical and mental health in patients at risk for or with neuropsychiatric disorders: considerations for clinical practice. Neuropsychiatr Dis Treat 2020;16:55-70.

47 WHO. Management of physical health conditions in adults with severe mental disorders: who guidelines 2018.

48 Gluth A, White D, Ward M. Lifestyle interventions in patients with serious mental illness. Lifestyle in Heart Health and Disease: Elsevier, 2018: 247-53.

49 Parker AG, Bailey AP. Exercise for adolescents and young people with mental illness. Exercise-Based Interventions for Mental Illness: Elsevier, 2018: 149-67.

50 De Rosa C, Sampogna G, Luciano M, et al. Improving physical health of patients with severe mental disorders: a critical review of lifestyle psychosocial interventions. Expert Rev Neurother 2017:17:667-81.

51 Rönngren Y, Björk A, Audulv Åsa, et al. Educational nurse-led lifestyle intervention for persons with mental illness. Int $J$ Ment Health Nurs 2018;27:1022-31.

52 Fiorillo A, Luciano M, Pompili M, et al. Editorial: reducing the mortality gap in people with severe mental disorders: the role of lifestyle psychosocial interventions. Front Psychiatry 2019;10:434

53 Taylor $\mathrm{Cl}$, Tompsett C, Sanders R, et al. The effectiveness of structured exercise programmes on psychological and physiological outcomes for patients with psychotic disorders: a systematic review and meta-analysis. International Journal of Sport and Exercise Psychology 2020;18:336-61.

54 Goracci A, Rucci P, Forgione RN, et al. Development, acceptability and efficacy of a standardized healthy lifestyle intervention in recurrent depression. J Affect Disord 2016;196:20-31.

55 Murphy JA, Oliver G, Ng CH, et al. Pilot-Testing of "Healthy Body Healthy Mind": An Integrative Lifestyle Program for Patients With a Mental Illness and Co-morbid Metabolic Syndrome. Front Psychiatry 2019;10:91

56 Bersani FS, Biondi M, Coviello M, et al. Psychoeducational intervention focused on healthy living improves psychopathological severity and lifestyle quality in psychiatric patients: preliminary findings from a controlled study. J Ment Health 2017;26:271-5.

57 Gurusamy J, Gandhi S, Damodharan D, et al. Exercise, diet and educational interventions for metabolic syndrome in persons with schizophrenia: a systematic review. Asian J Psychiatr 2018;36:73-85

58 Rosenbaum S, Newby JM, Steel Z, et al. Online physical activity interventions for mental disorders: a systematic review. Internet Interv 2015;2:214-20.

59 Deenik J, Tenback DE, Tak ECPM, et al. Changes in physical and psychiatric health after a multidisciplinary lifestyle enhancing treatment for inpatients with severe mental illness: the multi study I. Schizophr Res 2019;204:360-7.

60 Lambert JD, Greaves CJ, Farrand P, et al. Web-Based intervention using behavioral activation and physical activity for adults with depression (the eMotion study): pilot randomized controlled trial. $J$ Med Internet Res 2018;20:e10112.

61 Parker AG, Markulev C, Rickwood DJ, et al. Improving mood with physical activity (impact) trial: a cluster randomised controlled trial to determine the effectiveness of a brief physical activity behaviour change intervention on depressive symptoms in young people, compared with psychoeducation, in addition to routine clinical care within youth mental health services-a protocol study. BMJ Open 2019;9:e034002.

62 Nasstasia Y, Baker AL, Halpin SA, et al. Evaluating the efficacy of an integrated motivational interviewing and multi-modal exercise intervention for youth with major depression: healthy body, healthy mind randomised controlled trial protocol. Contemp Clin Trials Commun 2018:9:13-22.

63 Parker AG, Hetrick SE, Jorm AF, et al. The effectiveness of simple psychological and physical activity interventions for high prevalence mental health problems in young people: a factorial randomised controlled trial. J Affect Disord 2016;196:200-9.

64 Robillard R, Hermens D, Naismith S, et al. Ambulatory sleep-wake patterns and variability in young people with emerging mental disorders. J Psychiatry Neurosci. In Press 2015;40:28-37.

65 Harvey AG, Mullin BC, Hinshaw SP. Sleep and circadian rhythms in children and adolescents with bipolar disorder. Dev Psychopathol 2006:18:1147-68

66 Cousins JC, Whalen DJ, Dahl RE, et al. The bidirectional association between daytime affect and nighttime sleep in youth with anxiety and depression. J Pediatr Psychol 2011;36:969-79.

67 Minassian A, Henry BL, Geyer MA, et al. The quantitative assessment of motor activity in mania and schizophrenia. $J$ Affect Disord 2010;120:200-6.

68 Rohleder C, Song YJC, Crouse JJ, et al. Youth mental health Tracker: protocol to establish a longitudinal cohort and research database for young people attending Australian mental health services. BMJ Open 2020;10:e035379.

69 American Psychiatric AssociationArlington VA, ed. Diagnostic and statistical manual of mental disorders. 5, 2013.

70 Overall JE, Gorham DR. The brief psychiatric rating scale. Psychol Rep 1962;10:799-812.

71 Bell M, Milstein R, Beam-Goulet J, et al. The positive and negative syndrome scale and the brief psychiatric rating scale. reliability, comparability, and predictive validity. $J$ Nerv Ment Dis 1992;180:723-8.

72 Hickie IB, Scott EM, Hermens DF, et al. Applying clinical staging to young people who present for mental health care. Early Interv Psychiatry 2013;7:31-43.

73 McGorry PD, Hickie IB, Yung AR, et al. Clinical staging of psychiatric disorders: a heuristic framework for choosing earlier, safer and more effective interventions. Aust N Z J Psychiatry 2006;40:616-22.

74 Scott J, Leboyer M, Hickie I, et al. Clinical staging in psychiatry: a cross-cutting model of diagnosis with heuristic and practical value. Br J Psychiatry 2013;202:243-5.

75 Hickie IB, Scott J, McGorry PD. Clinical staging for mental disorders: a new development in diagnostic practice in mental health. Med J Aust 2013;198:461-2.

76 Hickie IB, Hermens DF, Naismith SL, et al. Evaluating differential developmental trajectories to adolescent-onset mood and psychotic disorders. BMC Psychiatry 2013;13:303.

77 Busner J, Targum SD. The clinical global impressions scale: applying a research tool in clinical practice. Psychiatry 2007;4:28-37.

78 Young RC, Biggs JT, Ziegler VE, et al. A rating scale for mania: reliability, validity and sensitivity. Br J Psychiatry 1978;133:429-35.

79 Rosenbaum S, Ward PB, International Working Group. The simple physical activity questionnaire. Lancet Psychiatry 2016;3:e1

80 Rosenbaum S, Morell R, Abdel-Baki A, et al. Assessing physical activity in people with mental illness: 23-country reliability and validity of the simple physical activity questionnaire (SIMPAQ). BMC Psychiatry 2020;20:1-12.

81 Yung AR, Yuen HP, McGorry PD, et al. Mapping the onset of psychosis: the comprehensive assessment of at-risk mental states. Aust N Z J Psychiatry 2005;39:964-71.

82 Hilsenroth MJ, Ackerman SJ, Blagys MD, et al. Reliability and validity of DSM-IV axis V. Am J Psychiatry 2000;157:1858-63.

83 Hay P, Katsikitis M, Begg J, et al. A two-year follow-up study and prospective evaluation of the DSM-IV axis V. Psychiatr Serv 2003:54:1028-30. 
84 Kessler RC, Andrews G, Colpe LJ, et al. Short screening scales to monitor population prevalences and trends in non-specific psychological distress. Psychol Med 2002;32:959-76.

85 Andrews $\mathrm{G}$, Slade T. Interpreting scores on the Kessler psychological distress scale (K10). Aust N Z J Public Health 2001;25:494-7.

86 Smout MF. The factor structure and predictive validity of the Kessler psychological distress scale (K10) in children and adolescents. Aust Psychol 2019;54:102-13.

87 Craig CL, Marshall AL, Sjöström M, et al. International physical activity questionnaire: 12 -country reliability and validity. Med Sci Sports Exerc 2003;35:1381-95.

88 Booth M. Assessment of physical activity: an international perspective. Res Q Exerc Sport 2000;71:114-20.

89 Berryman C, McAuley JH, Moseley LG. Sphere 12 screening questionnaire. J Physiother 2012;58:273.

90 Smyth C. The Pittsburgh sleep quality index (PSQI). J Gerontol Nurs 1999;25:10

91 Shahid A, Wilkinson K, Marcu S. Munich Chronotype questionnaire (MCTQ). stop. THAT and One Hundred Other Sleep Scales: Springer, 2011: 245-7.

92 Raniti MB, Waloszek JM, Schwartz O, et al. Factor structure and psychometric properties of the Pittsburgh sleep quality index in community-based adolescents. Sleep 2018;41:zsy066.

93 Carpenter JS, Andrykowski MA. Psychometric evaluation of the Pittsburgh sleep quality index. J Psychosom Res 1998;45:5-13.

94 Insomnia: psychological assessment and management, 1993. Guilford press

95 Morin CM, Belleville G, Bélanger L, et al. The insomnia severity index: psychometric indicators to detect insomnia cases and evaluate treatment response. Sleep 2011;34:601-8.

96 van Spijker BAJ, Batterham PJ, Calear AL, et al. The suicidal ideation attributes scale (SIDAS): community-based validation study of a new scale for the measurement of suicidal ideation. Suicide Life Threat Behav 2014;44:408-19.

97 Rush AJ, Trivedi MH, Ibrahim HM, et al. The 16-Item quick inventory of depressive symptomatology (QIDS), clinician rating (QIDS-C), and self-report (QIDS-SR): a psychometric evaluation in patients with chronic major depression. Biol Psychiatry 2003;54:573-83.

98 Norman SB, Cissell SH, Means-Christensen AJ, et al. Development and validation of an overall anxiety severity and impairment scale (OASIS). Depress Anxiety 2006;23:245-9.

99 Humeniuk R, Ali R, Babor TF, et al. Validation of the alcohol, smoking and substance involvement screening test (assist). Addiction 2008;103:1039-47.

100 Group WHOAW. The alcohol, smoking and substance involvement screening test (assist): development, reliability and feasibility. Addiction 2002;97:1183-94.

101 Newcombe DA, Humeniuk RE, Ali R. Validation of the world Health organization alcohol, smoking and substance involvement screening test (assist): report of results from the Australian site. Drug Alcohol Rev 2005;24:217-26.

102 Bush K, Kivlahan DR, McDonell MB, et al. The audit alcohol consumption questions (AUDIT-C): an effective brief screening test for problem drinking. ambulatory care quality improvement project (ACQUIP). alcohol use disorders identification test. Arch Intern Med 1998;158:1789-95.

103 de Meneses-Gaya C, Zuardi AW, Loureiro SR, et al. Alcohol use disorders identification test (audit): an updated systematic review of psychometric properties. Psychol Neurosci 2009;2:83-97.

104 Hay PJ, Mond J, Buttner P, et al. Eating disorder behaviors are increasing: findings from two sequential community surveys in South Australia. PLoS One 2008;3:e1541.

105 Mitchison D, Hay P, Slewa-Younan S, et al. The changing demographic profile of eating disorder behaviors in the community. BMC Public Health 2014;14:943.

106 Berg KC, Peterson CB, Frazier P, et al. Psychometric evaluation of the eating disorder examination and eating disorder examinationquestionnaire: a systematic review of the literature. Int $J$ Eat Disord 2012;45:428-38.

107 Rosenberg M. Acceptance and commitment therapy Measures package. In: Rosenberg self-esteem scale (Rse). , 1965: 61, 18.

108 Robins RW, Hendin HM, Trzesniewski KH. Measuring global selfesteem: construct validation of a single-item measure and the Rosenberg self-esteem scale. Personality and Social Psychology Bulletin 2001;27:151-61.

109 Bagley C, Mallick K. Normative data and mental health construct validity for the Rosenberg self-esteem scale in British adolescents. Int $J$ Adolesc Youth 2001;9:117-26.

110 Larsen DL, Attkisson CC, Hargreaves WA, et al. Assessment of client/patient satisfaction: development of a general scale. Eval Program Plann 1979;2:197-207.

111 Hill NR, Levy JC, Matthews DR. Expansion of the homeostasis model assessment of $\beta$-cell function and insulin resistance to enable clinical trial outcome modeling through the interactive adjustment of physiology and treatment effects: iHOMA2. Diabetes Care 2013;36:2324-30.

112 Difrancesco S, Lamers F, Riese $\mathrm{H}$, et al. Sleep, circadian rhythm, and physical activity patterns in depressive and anxiety disorders: a 2-week ambulatory assessment study. Depress Anxiety 2019;36:975-86.

113 Schaefer CA, Nigg CR, Hill JO, et al. Establishing and evaluating wrist cutpoints for the GENEActiv accelerometer in youth. Med Sci Sports Exerc 2014;46:826-33.

114 Hildebrand M, VAN Hees VT, Hansen BH, et al. Age group comparability of raw accelerometer output from wrist- and hip-worn monitors. Med Sci Sports Exerc 2014;46:1816-24.

115 Esliger DW, Rowlands AV, Hurst TL, et al. Validation of the GENEA Accelerometer. Med Sci Sports Exerc 2011;43:1085-93.

116 van Hees VT, Gorzelniak L, Dean León EC, et al. Separating movement and gravity components in an acceleration signal and implications for the assessment of human daily physical activity. PLoS One 2013;8:e61691.

117 te Lindert BHW, Van Someren EJW. Sleep estimates using microelectromechanical systems (MEMS). Sleep 2013;36:781-9.

118 van Hees VT, Sabia S, Anderson KN, et al. A novel, open access method to assess sleep duration using a Wrist-Worn Accelerometer. PLoS One 2015;10:e0142533. 